\title{
Dark solitons and their head-on collisions in Bose-Einstein condensates
}

\author{
Guoxiang Huang, ${ }^{1,2}$ Manuel G. Velarde, ${ }^{2}$ and Valeri A. Makarov ${ }^{2}$ \\ ${ }^{1}$ Key Laboratory of Optical and Magnetic Resonance Spectroscopy and Department of Physics, East China Normal University, \\ Shanghai 200062, China \\ ${ }^{2}$ Instituto Pluridisciplinar, Universidad Complutense de Madrid, Paseo Juan XXIII, No. 1, Madrid 28040, Spain
}

(Received 19 May 2000; revised manuscript received 20 February 2001; published 8 June 2001)

\begin{abstract}
The evolution and collision of dark solitary waves (solitons) appearing in cigar-shaped Bose-Einstein condensates with repulsive atom-atom interaction are here considered using a Boussinesq-Korteweg-de Vries description. We provide theoretical predictions and computer experiment evidence about their phase shifts or change of trajectories, in the space-time plot, corresponding upon collisions. Details are also given about a suggested experiment that could assess their genuine solitonic nature.
\end{abstract}

DOI: 10.1103/PhysRevA.64.013617

PACS number(s): 03.75.Fi, 03.65.Ge

\section{INTRODUCTION}

The remarkable experimental realization of the BoseEinstein condensation in trapped clouds of alkali-atoms $[1-4]$ has stimulated experimental and theoretical investigations on the physics of dilute ultracold bosonic gases [5-7]. One of the important aspects in this area is the exploration of nonlinear properties of matter waves. Nonlinear excitations, such as vortices and solitons, have been observed in BoseEinstein condensates (BEC's) [8-10] and the four-wave mixing has also been recently realized. [11] These studies are significant for nonlinear atom optics and for other areas of condensed-matter physics and fluid dynamics [12-21].

In a BEC, in addition to the atom-atom interaction, all atoms move in an external trap potential, i.e., the system is inhomogeneous, and the behavior of the solitons is very rich. For the BEC's with rubidium and sodium atomic vapors in a trap, the solitary excitations in the condensates are of the dark type since the interaction between atoms is repulsive. In recent experiments by Burger et al. [9] and by Denschlag et al. [10], dark solitons were generated by a phase imprinting method in a cigar-shaped BEC of ${ }^{87} \mathrm{Rb}$ and in a nearly spherical BEC of ${ }^{23} \mathrm{Na}$, respectively. In both experiments an interesting phenomenon was found, i.e., in addition to a density minimum of the condensate traveling at lower velocity than the speed of sound, which is identified as subsonic or subcritically moving dark soliton, another density minimum traveling in opposite direction may appear. Multidark solitons were also created by applying higher imprinted phase values. These findings imply that one can observe a collision between two dark solitons in BEC's, and hence, the possibility of experimentally assessing their genuine solitonic nature $[20,21]$.

On the other hand, it seems interesting to further extend the available theory on solitons in BEC's [22-34] with a repulsive atom-atom interaction. Recently, an interesting new approach has been proposed [35] for the soliton propagation in a cigar-shaped BEC. In this approach, besides a Thomas-Fermi (TF) approximation for the ground state and also for the nonlinear excitations, the assumption of the existence of an interface (elastic cylindrical shell) for the condensate is also made. The interface is further assumed to obey Newton's second law. Under these assumptions, a
Boussinesq-Korteweg-de Vries (B-KdV) equation [36] was derived using a reductive perturbation method if the excitation from the condensate is weakly nonlinear. Instead of a dark soliton, a supercritical bump (i.e., density maximum) soliton is obtained, hence traveling supersonically [35]. Such a result is at variance with those obtained in most of the studies, using different approaches [22-34] for solitons in BEC's with repulsive interactions. Since in the experiments of Burger et al. [9] and Denschlag et al. [10], the solitons observed are small-amplitude gray ones, which belong to the type of weakly nonlinear excitations on a condensate background, it is reasonable to use a $\mathrm{B}-\mathrm{KdV}$ theory to describe such weakly nonlinear excitations. On the other hand, in the scheme of the B-KdV description, the collision property of the solitons is well established [19-21]. Thus, it appears necessary to reconsider the above-mentioned description to find out the reason of the indicated inconsistency and study the colliding property of the solitons in BEC's in detail.

In this paper, we investigate the dynamics of solitons in a cigar-shaped BEC by developing a B-KdV description without using the TF approximation. Then we pay attention to the head-on collision between two dark solitons in the BEC and give their expected change of trajectories, in the corresponding space-time plot, or phase shifts upon collision. The paper is organized as follows. In Sec. II, starting from the GrossPitaevskii (GP) equation, a B-KdV equation is derived by a multiple-scales method without using the TF approximation. The dark-soliton solutions are also provided and discussed. A head-on collision between two dark solitons traveling in opposite directions is considered in Sec. III. The phase shift of each soliton due to the collision is explicitly calculated using the Poincaré-Lighthill-Kuo (PLK) method [37]. In Sec. IV we report the results of our numerical investigation of soliton propagation and collisions. Finally, Sec. V contains a discussion and summary of our results.

\section{A B-KDV DESCRIPTION FOR DARK SOLITON EXCITATIONS}

The dynamic behavior of interacting bosonic gases at zero temperature is well described by the time-dependent GrossPitaevskii (GP) equation for the order parameter [6] 


$$
i \hbar \frac{\partial \Psi}{\partial t}=\left[-\frac{\hbar^{2}}{2 m} \nabla^{2}+V_{\text {ext }}(\mathbf{r})+g|\Psi|^{2}\right] \Psi,
$$

where $\int d \mathbf{r}|\Psi|^{2}=N$ is the number of atoms in the condensate, $g=4 \pi \hbar^{2} a / m$ is the interacting constant with $m$ the mass of the atom, and $a$ the s-wave scattering length $(a>0$ for repulsive interactions). As in experiment [9], we consider a cigar-shaped harmonic trap with the elongated axis in the $x$ direction. Thus, we have $V_{\text {ext }}(\mathbf{r})=(m / 2)\left[\omega_{x}^{2} x^{2}+\omega_{\perp}^{2}\left(y^{2}\right.\right.$ $\left.\left.+z^{2}\right)\right]$ with $\omega_{x} \ll \omega_{\perp}$, where $\omega_{x}$ and $\omega_{\perp}$ are the frequencies of the trap in the $x$ direction and the transverse direction, respectively. Expressing the order parameter in terms of its modulus and phase, $\Psi=\sqrt{n} \exp (i \phi)$, we obtain a set of coupled equations for $n$ and $\phi$. By introducing $(x, y, z)$ $=a_{\perp}\left(x^{\prime}, y^{\prime}, z^{\prime}\right), t=\omega_{\perp}^{-1} t^{\prime}, n=n_{0} n^{\prime}$ with $a_{\perp}=\sqrt{\hbar /\left(m \omega_{\perp}\right)}$ and $n_{0}=N / a_{\perp}^{3}$, we have the following dimensionless equations of motion (after dropping the primes, expecting no confusion in the reader):

$$
\begin{gathered}
\frac{\partial n}{\partial t}+\nabla \cdot(n \boldsymbol{\nabla} \phi)=0 \\
\frac{\partial \phi}{\partial t}+\frac{1}{2}\left(\frac{\omega_{x}}{\omega_{\perp}}\right)^{2} x^{2}+\frac{1}{2}\left(y^{2}+z^{2}\right)+Q n \\
+\frac{1}{2}\left[(\nabla \phi)^{2}-\frac{1}{\sqrt{n}} \nabla^{2} \sqrt{n}\right]=0,
\end{gathered}
$$

with $Q=4 \pi N a / a_{\perp}, \nabla=(\partial / \partial x, \partial / \partial y, \partial / \partial z)$ and $\int d \mathbf{r} n=1$. The last term on the left-hand side of Eq. (3) (i.e., $\left.-1 / 2 \sqrt{n} \nabla^{2} \sqrt{n}\right)$ is called the quantum pressure, which provides the dispersion necessary to form a dark soliton in the BEC, as we shall see below.

Equations (2) and (3) are (3+1)-dimensional, nonlinear, and dispersive equations with a variable coefficient, and we have not yet been able to find an exact solution. For this reason we turned to an effective approach with some reasonable approximations. Since in experiment [9], $\omega_{x} / \omega_{\perp}$ is small ( $\approx 0.03$ ), one can expect that the variation of the profile of the order parameter is slow in the elongated (i.e., $x$ ) direction. On other hand, due to the strong confinement in the transverse directions, the motion of the order parameter in the $x$ and $y$ directions behaves like a standing wave, i.e., the system is similar to a wave guide in which the excitation from the ground state propagates in the elongated direction $[23,27,35,38]$. The strong confinement also ensures the dynamical stability of a dark soliton excitation [27]. Therefore, we assume $\sqrt{n}=A(x, t) G(y, z)$ and $\phi=-\mu t+\varphi(x, t)$. Then $G(y, z)$ satisfies

$$
-\frac{1}{2}\left(\frac{\partial^{2}}{\partial y^{2}}+\frac{\partial^{2}}{\partial z^{2}}\right) G+\frac{1}{2}\left(y^{2}+z^{2}\right) G=\nu G .
$$

Equation (4) is the eigenvalue problem of a two-dimensional quantum harmonic oscillator. Its ground-state solution is $G_{0}(y, z)=\exp \left[-\left(y^{2}+z^{2}\right) / 2\right]$ with the eigenvalue $\nu=\nu_{0}=1$. With these substitutions, Eqs. (2) and (3) are transformed into

$$
\begin{gathered}
\frac{\partial A}{\partial t}+\frac{\partial A}{\partial x} \frac{\partial \varphi}{\partial x}+\frac{1}{2} A \frac{\partial^{2} \varphi}{\partial x^{2}}=0, \\
-\frac{1}{2} \frac{\partial^{2} A}{\partial x^{2}}+\frac{1}{2}\left(\frac{\omega_{x}}{\omega_{\perp}}\right)^{2} x^{2} A+(-\mu+1) A \\
+\left[\frac{\partial \varphi}{\partial t}+\frac{1}{2}\left(\frac{\partial \varphi}{\partial x}\right)^{2}\right] A+\frac{1}{2} Q A^{3}=0 .
\end{gathered}
$$

As in Ref. [38], to obtain Eq. (6) we have used Eq. (4) with $G=G_{0}$ and multiplied Eq. (3) by $G_{0}^{*}$ and then integrated once with respect to $y$ and $z$. This procedure is equivalent to assume that the excitation is quasi-one-dimensional $[23,31,35,38,39]$.

Let $A=u_{0}+a(x, t)$ (without loss of generality, we assume that $u_{0}$, a quantity characterizing the condensate background, is positive) with $(a, \varphi)=\left(a_{0}, \varphi_{0}\right) \exp [i(k x-\omega t)]+c . c$. with $u_{0}, a_{0}$, and $\varphi_{0}$ constants, when the trapping potential in the $x$ direction is neglected, we obtain the linear dispersion relation of Eqs. (5) and (6)

$$
\omega= \pm \frac{1}{2} k\left(2 Q u_{0}^{2}+k^{2}\right)^{1 / 2},
$$

where the positive (respectively, negative) sign corresponds to the wave propagating to the right (respectively, left). We stress that the $k^{2}$-term in the bracket of Eq. (7) comes from the quantum pressure, given by the term $-(1 / 2) \partial^{2} A / \partial x^{2}$ in Eq. (6). Equation (7) is the Bogoliubov-type linear excitation spectrum of our system for sound propagation. We see that to get the Bogoliubov excitation spectrum, the quantum pressure of the system plays a significant role. From Eq. (7) we obtain the sound speed of the system $c=\left.(d \omega / d k)\right|_{k=0}$ $= \pm \sqrt{Q / 2} u_{0}$. For an homogeneous system [i.e., $V_{\text {ext }}(\mathbf{r})$ $=0]$ the corresponding sound speed is $c_{0}= \pm \sqrt{Q} u_{0}$ in our notation. Thus, we have $c / c_{0}=1 / \sqrt{2}$. The factor $1 / \sqrt{2}$ is due to the transverse confinement of the system. This result is consistent with the experiment by Andrews et al. [40] and the theoretical approaches in Ref. [23] for sound propagation in BECs with repulsive atom-atom interactions. In addition, we see that the wave may propagate in two opposite directions and displays dispersion. This is also the case observed in the experiment [40].

Now we consider the weakly nonlinear excitations on the condensate background (i.e., the ground state) of the system. We note that in the experiment of Burger et al. [9] the sound speed is $c=\sqrt{4 \pi a n_{0}} \hbar / m \approx 3.7 \mathrm{~mm} / \mathrm{s}$, where $n_{0}$ is the density of the condensate. Thus, the healing length $l_{0}=1 / \sqrt{4 \pi a n_{0}}$ is about $0.2 \mu \mathrm{m}$. For a gray soliton, with grayness [see Eq. (15) below] $\widetilde{A}_{0}=0.1$, its width is about $0.9 \mu \mathrm{m}$. Hence, their width may be larger than the healing length, and the size of the gray solitons may be large enough for easy observation. The harmonic oscillator length in the elongated direction in Ref. [9] is about $3 \mu \mathrm{m}$, which is much larger than the healing length of the system. This means that the variation of the order parameter is slow in the $x$ direction, as mentioned above. For simplicity in the analytical approach, we thus 
neglect the second term on the left side of Eq. (6) (we shall discuss the effect of the inhomogeneity in the $x$ direction in Sec. IV).

Using the asymptotic expansion

$$
\begin{gathered}
A=u_{0}+\epsilon^{2}\left[a^{(0)}+\epsilon^{2} a^{(1)}+\cdots\right], \\
\varphi=\epsilon\left[\varphi^{(0)}+\epsilon^{2} \varphi^{(1)}+\cdots\right],
\end{gathered}
$$

and assuming that $a^{(j)}$ and $\varphi^{(j)}(j=0,1, \cdots)$ are functions of the multiple-scale variables $\xi=\epsilon(x-c t)$ and $\tau=\epsilon^{3} t$, where $\epsilon$ is a smallness parameter characterizing the relative amplitude of the excitation, and then substituting them to Eqs. (5) and (6), we obtain

$$
\begin{gathered}
c \frac{\partial a^{(j)}}{\partial \xi}-\frac{1}{2} u_{0} \frac{\partial^{2} \varphi^{(j)}}{\partial \xi^{2}}=\alpha^{(j)}, \\
Q u_{0}^{2} a^{(j)}-c u_{0} \frac{\partial \varphi^{(j)}}{\partial \xi}=\beta^{(j)} .
\end{gathered}
$$

The explicit expressions of $\alpha^{(j)}$ and $\beta^{(j)}(j=0,1, \cdots)$ are not needed here.

In the leading order $(j=0)$, we obtain $\varphi^{(0)}$ $=\left(2 c / u_{0}\right) \int d \xi a^{(0)}$ with $a^{(0)}$ a function yet to be determined. The solvability condition demands $c=\delta_{1} \sqrt{Q / 2} u_{0}$ with $\delta_{1}$ $= \pm 1$. At the next order $(j=1)$, the solvability condition results in the closed equation for $a^{(0)}$ :

$$
\frac{\partial a^{(0)}}{\partial \tau}+\frac{3 c}{u_{0}} a^{(0)} \frac{\partial a^{(0)}}{\partial \xi}-\frac{1}{8 c} \frac{\partial^{3} a^{(0)}}{\partial \xi^{3}}=0 .
$$

Equation (12) is the B-KdV equation for the wave traveling to the right (left) for the case $\delta_{1}=+1\left(\delta_{1}=-1\right)$. Note that we refer to disturbances upon the pedestal profile offered by the cigar-shaped background condensate and that according to the signs in Eq. (12), we can consider either $t \rightarrow \infty$ or $t$ $\rightarrow-\infty$ for evolutionary purposes. Note also that the dispersion term in Eq. (12) is due to the contribution of the quantum pressure of Eq. (3). Let $w=\epsilon^{2} a^{(0)}$ and use the definition of $\xi$ and $\tau$. Then we obtain

$$
\frac{\partial w}{\partial t}+\frac{3 c}{u_{0}} w \frac{\partial w}{\partial X}-\frac{1}{8 c} \frac{\partial^{3} w}{\partial X^{3}}=0
$$

with $X=x-c t$. The single-soliton solution of Eq. (13) is given by

$$
\begin{aligned}
w & =-A_{0} \operatorname{sech}^{2}\left[\sqrt{\frac{2 c^{2} A_{0}}{u_{0}}}\left(X+c \frac{A_{0}}{u_{0}} t-x_{0}\right)\right] \\
& =-A_{0} \operatorname{sech}^{2}\left[\sqrt{\frac{2 c^{2} A_{0}}{u_{0}}}\left\{x-c\left(1-\frac{A_{0}}{u_{0}}\right) t-x_{0}\right\}\right],
\end{aligned}
$$

where $A_{0}$ is a positive constant, $x_{0}$ is a constant denoting the initial position of the soliton on the pedestal background. Exact to the first order, the condensed-state wave function takes the form

$$
\begin{aligned}
\Psi= & u_{0}\left(1-\widetilde{A}_{0} \operatorname{sech}^{2}\left[\sqrt{2 c^{2} \widetilde{A}_{0}}\left\{x-c\left(1-\widetilde{A}_{0}\right) t-x_{0}\right\}\right]\right) \\
& \times \exp \left[-\frac{y^{2}+z^{2}}{2}\right] \exp [i(-\mu t+\varphi)],
\end{aligned}
$$

with $\widetilde{A}_{0}=A_{0} / u_{0}, \mu=1+Q u_{0}^{2} / 2$. The constant $\widetilde{A}_{0}$ can be taken as the grayness of the soliton. The phase function reads

$$
\varphi=-\sqrt{2 \widetilde{A}_{0}} \tanh \left[\sqrt{2 c^{2} \widetilde{A}_{0}}\left\{x-c\left(1-\widetilde{A}_{0}\right) t-x_{0}\right\}\right] .
$$

Let us emphasize the results obtained above:

(i) From Eq. (15) we see that the excitation is a dark soliton (i.e., density minimum of the condensate), consistent with most of the studies on soliton dynamics in BEC with a repulsive interaction using different approaches [23-25,3134].

(ii) The velocity of the dark soliton is $v_{s}=c\left(1-\widetilde{A}_{0}\right)$ hence directly dependent on the depth of the depression below the background pedestal level. Accordingly, the deeper this depth, the lower is the soliton velocity. Thus, $v_{s}$ is always lower than the sound speed $c$ of the system (see also the result in Ref. [25]). This ensures its stability and is consistent with known results for dark solitons. In fact, it is just this property that makes Burger et al. [9] and Denschlag et al. [10] to identify the density minima in their BEC experiments as dark solitons rather than mere sound waves.

(iii) The formation of the dark soliton given in Eq. (15) is due to the balance between the nonlinearity and the dispersion in Eq. (12). Consequently, the quantum pressure plays a significant role in the formation of the dark soliton in the BEC, in agreement with the results obtained in Refs. $[23,25,31]$.

(iv) From Eq. (16) we obtain

$$
\left|\frac{\partial \varphi}{\partial x}\right|=2 c \widetilde{A}_{0} \operatorname{sech}^{2}\left[\sqrt{2 c^{2} \widetilde{A}_{0}}\left\{x-c\left(1-\widetilde{A}_{0}\right) t-x_{0}\right\}\right] .
$$

It reveals that, indeed, when the phase gradient is increased, the soliton becomes darker and indeed travels slower; when the phase gradient is lowered, the soliton becomes shallower and propagates faster, agreeing with the observation in the experiments $[9,10]$.

(v) The velocity of the dark soliton may have two different signs $\left[v_{s}= \pm \sqrt{Q / 2} u_{0}\left(1-\widetilde{A}_{0}\right)\right]$, i.e., it may propagate in either the positive or the negative $x$ direction. Recall that, as we noted earlier, in view of the signs in Eq. (12) we can also consider $t \rightarrow-\infty$ rather than $t \rightarrow \infty$. This may provide a possible explanation of the appearance of two density minima (i.e., two dark solitons) traveling in opposite directions, observed in the experiments by Burger et al. [9] and by Denschlag et al. [10] 


\section{HEAD-ON COLLISIONS OF TWO DARK SOLITONS}

In a one- (or quasi-one-) dimensional system, there are two distinct soliton interactions. One is the overtaking collision and the other one is the head-on collision [41-46]. The overtaking collision of the dark solitons in the BEC can be studied with the B-KdV Eq. (12). Its multisoliton solutions (they travel in the same direction) can be obtained from the inverse scattering transform [21]. However, for the head-on collision, we must search for the evolution of waves traveling to both sides, and hence, we need to employ a suitable asymptotic expansion to solve the original equations of motion (2) and (3), or their simplified form (5) and (6).

Starting from Eqs. (5) and (6), we assume that in the condensate, two dark solitons are generated that are, asymptotically, far apart at the initial state and proceed toward each other. After some time they interact, collide, and then depart. We use the PLK method $[37,43,45,46]$ to investigate the head-on collision between two dark solitons in the BEC. We anticipate that the collision will result in a change of trajectory or phase shift for each soliton, and hence, assume

$$
\begin{aligned}
& \xi=\epsilon\left(x-c_{R} t\right)+\epsilon^{2} P^{(0)}(\eta)+\epsilon^{4} P^{(1)}(\xi, \eta)+\cdots, \\
& \eta=\epsilon\left(x+c_{L} t\right)+\epsilon^{2} Q^{(0)}(\eta)+\epsilon^{4} Q^{(1)}(\xi, \eta)+\cdots
\end{aligned}
$$

which denote the trajectories of the solitons traveling to the right and left, respectively. In Eqs. (18) and (19) the rightand left-running wave velocities $c_{R}$ and $c_{L}$ are related to the amplitudes of the waves. The functions $P^{(j)}$ and $Q^{(j)}(j$ $=0,1,2, \ldots$,$) are yet to be determined. The aim of intro-$ ducing these functions is to make a uniformly valid asymptotic expansion (i.e., to eliminate secular terms) and at the same time obtain the change of the trajectories (i.e., phase shifts) of the solitons after the collision. Using Eqs. (18) and (19), we get the transformation between derivatives as

$$
\begin{gathered}
\frac{\partial}{\partial x}=\epsilon\left(\frac{\partial}{\partial \xi}+\frac{\partial}{\partial \eta}\right)+\epsilon^{3}\left(\frac{\partial P^{(0)}}{\partial \eta} \frac{\partial}{\partial \xi}+\frac{\partial Q^{(0)}}{\partial \xi} \frac{\partial}{\partial \eta}\right)+\cdots \\
\frac{\partial}{\partial t}=\epsilon\left(-c_{R} \frac{\partial}{\partial \xi}+c_{L} \frac{\partial}{\partial \eta}\right)+\epsilon^{3}\left(c_{L} \frac{\partial P^{(0)}}{\partial \eta} \frac{\partial}{\partial \xi}-c_{R} \frac{\partial Q^{(0)}}{\partial \xi} \frac{\partial}{\partial \eta}\right) \\
+\cdots .
\end{gathered}
$$

Introducing the same asymptotic expansions for the quantities $A$ and $\varphi$ as in Eqs. (8) and (9) and assuming

$$
\begin{aligned}
& c_{R}=c+\epsilon^{2} R_{1}+\epsilon^{4} R_{2}+\cdots, \\
& c_{L}=c+\epsilon^{2} L_{1}+\epsilon^{4} L_{2}+\cdots,
\end{aligned}
$$

and then substituting Eqs. (20)-(23) into Eqs. (5) and (6), we obtain

$$
c\left(\frac{\partial}{\partial \xi}-\frac{\partial}{\partial \eta}\right) a^{(j)}-\frac{1}{2} u_{0}\left(\frac{\partial}{\partial \xi}+\frac{\partial}{\partial \eta}\right)^{2} \varphi^{(j)}=M^{(j)},
$$

$$
Q u_{0}^{2} a^{(j)}-c u_{0}\left(\frac{\partial}{\partial \xi}-\frac{\partial}{\partial \eta}\right) \varphi^{(j)}=N^{(j)}
$$

The expressions for $M^{(j)}$ and $N^{(j)}(j=0,1,2, \ldots$,$) are not$ needed here.

The leading order solution $(j=0)$ of Eqs. (24) and (25) reads

$$
\begin{gathered}
a^{(0)}=f_{0}(\xi)+g_{0}(\eta), \\
\varphi^{(0)}=\frac{2 c}{u_{0}}\left(\int_{+\infty}^{\xi} f_{0}\left(\xi^{\prime}\right) d \xi^{\prime}-\int_{-\infty}^{\eta} g_{0}\left(\eta^{\prime}\right) d \eta^{\prime}\right),
\end{gathered}
$$

with a solvability condition $c=\sqrt{Q / 2} u_{0}$ (the sound speed). Thus, in the leading order we have two sound waves, $f_{0}(\xi)$ and $g_{0}(\eta)$, which are traveling to the right and to the left, respectively. The lower limits of the integrations in Eqs. (27) have been chosen to make the initial phases (before collision) of the soliton $f_{0}(\xi)$ and $g_{0}(\eta)$ equal to zero.

In the second order $(j=1)$, by Eqs. (24) and (25) one has the equations for $a^{(1)}$ and $\varphi^{(1)}$. The solvability conditions for $a^{(1)}$ and $\varphi^{(1)}$ yield

$$
\begin{gathered}
\frac{c A_{0}}{u_{0}} \frac{\partial f_{0}}{\partial \xi}+\frac{3 c}{u_{0}} f_{0} \frac{\partial f_{0}}{\partial \xi}-\frac{1}{8 c} \frac{\partial^{3} f_{0}}{\partial \xi^{3}}=0, \\
\frac{c B_{0}}{u_{0}} \frac{\partial g_{0}}{\partial \eta}+\frac{3 c}{u_{0}} g_{0} \frac{\partial g_{0}}{\partial \eta}-\frac{1}{8 c} \frac{\partial^{3} g_{0}}{\partial \eta^{3}}=0, \\
\frac{\partial P^{(0)}}{\partial \eta}=\frac{1}{2} g_{0}(\eta), \\
\frac{\partial Q^{(0)}}{\partial \xi}=\frac{1}{2} f_{0}(\xi)
\end{gathered}
$$

together with $R_{1}=-\left(c / u_{0}\right) A_{0}$ and $L_{1}=-\left(c / u_{0}\right) B_{0}$, where $A_{0}$ and $B_{0}$ are two positive constants related, respectively, to the one and other of the amplitudes. Equations (28) and (29) are the two-side traveling wave B-KdV equations in the reference frames of $\xi$ and $\eta$, respectively. Their corresponding solutions are

$$
f_{0}(\xi)=-A_{0} \operatorname{sech}^{2}\left[\sqrt{\frac{2 c^{2} A_{0}}{u_{0}} \xi}\right] \quad \text { (right-running soliton) }
$$

$$
g_{0}(\eta)=-B_{0} \operatorname{sech}^{2}\left[\sqrt{\frac{2 c^{2} B_{0}}{u_{0}} \eta}\right] \quad \text { (left-running soliton) }
$$

which describe depressions of the background pedestal level. Using Eqs. (30) and (31) we obtain

$$
P^{(0)}(\eta)=-\frac{1}{2 c} \sqrt{\frac{u_{0} B_{0}}{2}}\left(\tanh \left[\sqrt{\frac{2 c^{2} B_{0}}{u_{0}}} \eta\right]+1\right),
$$




$$
Q^{(0)}(\xi)=-\frac{1}{2 c} \sqrt{\frac{u_{0} A_{0}}{2}}\left(\tanh \left[\sqrt{\frac{2 c^{2} A_{0}}{u_{0}}} \xi\right]-1\right) .
$$

From Eqs. (32)-(35) we can obtain the expressions for $a^{(1)}$ and $\varphi^{(1)}$. Finally, the solution up to $O\left(\epsilon^{4}\right)$ order is

$$
\begin{aligned}
A= & u_{0}-\epsilon^{2}\left[A_{0} \operatorname{sech}^{2} \theta_{A}(\xi)+B_{0} \operatorname{sech}^{2} \theta_{B}(\eta)\right] \\
& +\epsilon^{4}\left[\frac{A_{0}^{2}}{u_{0}} \operatorname{sech}^{2} \theta_{A}(\xi)+\frac{B_{0}^{2}}{u_{0}} \operatorname{sech}^{2} \theta_{B}(\eta)\right. \\
& +\frac{3 A_{0}^{2}}{2 u_{0}} \operatorname{sech}^{4} \theta_{A}(\xi)+\frac{3 B_{0}^{2}}{2 u_{0}} \operatorname{sech}^{4} \theta_{B}(\eta) \\
& +\left(\frac{1}{u_{0}^{2}}-1\right) A_{0} B_{0} \operatorname{sech}^{2} \theta_{A}(\xi) \operatorname{sech}^{2} \theta_{B}(\eta) \\
& \left.+f_{1}(\xi)+g_{1}(\eta)\right], \\
\varphi=\epsilon & \left.\sqrt{\frac{2 B_{0}}{u_{0}}}\left[\tanh \theta_{B}(\eta)+1\right]-\sqrt{\frac{2 A_{0}}{u_{0}}}\left[\tanh \theta_{A}(\xi)-1\right]\right) \\
+ & \epsilon^{3} \frac{2 c}{u_{0}}\left(\int_{+\infty}^{\xi} f_{1}\left(\xi^{\prime}\right) d \xi^{\prime}-\int_{-\infty}^{\eta} g_{1}\left(\eta^{\prime}\right) d \eta^{\prime}\right)+O\left(\epsilon^{5}\right),
\end{aligned}
$$

where $\theta_{A}(\xi)=\sqrt{2 c^{2} A_{0} / u_{0}} \xi \quad$ and $\quad \theta_{B}(\eta)=\sqrt{2 c^{2} B_{0} / u_{0}} \eta$. $f_{1}(\xi)$ and $g_{1}(\eta)$ are two functions to be determined in the next order. The trajectories of the soliton $f_{0}(\xi)$ (denoted by A) and the soliton $g_{0}(\eta)$ (denoted by B) are, respectively, given by

$$
\begin{aligned}
& \xi=\epsilon\left(x-c_{R} t\right)-\epsilon^{2} \frac{1}{2 c} \sqrt{\frac{B_{0} u_{0}}{2}}\left[\tanh \theta_{B}(\eta)+1\right]+O\left(\epsilon^{4}\right), \\
& \eta=\epsilon\left(x+c_{L} t\right)-\epsilon^{2} \frac{1}{2 c} \sqrt{\frac{A_{0} u_{0}}{2}}\left[\tanh \theta_{A}(\xi)-1\right]+O\left(\epsilon^{4}\right),
\end{aligned}
$$

with $c_{R}=c\left(1-\epsilon^{2} A_{0} / u_{0}\right)+O\left(\epsilon^{4}\right)$ and $c_{L}=c\left(1-\epsilon^{2} B_{0} / u_{0}\right)$ $+O\left(\epsilon^{4}\right)$, and hence, both are subsonic solitary waves. Besides, once more we see that their velocities are drastically lowered as their corresponding depression depths increase.

The phase shifts after a head-on collision of the two dark solitons can be obtained using Eqs. (38) and (39). To do this, let us assume that the solitons $A$ and $B$ are (asymptotically) far from each other at the initial time $(t=-\infty)$; i.e., soliton $A$ is at $\xi=0, \eta=-\infty$, and soliton $B$ is at $\eta=0$ and $\xi=+\infty$, respectively. After the collision $(t=+\infty)$, the soliton $A$ is far to the right of soliton $B$, i.e., soliton $A$ is at $\xi=0, \eta=+\infty$, and soliton $B$ is at $\eta=0, \xi=-\infty$. Using Eqs. (38) and (39) we obtain their corresponding phase shifts $\Delta_{A}$ and $\Delta_{B}$ as follows:

$$
\begin{aligned}
\Delta_{A} & =\left.\epsilon\left(x-c_{R} t\right)\right|_{\xi=0, \eta=+\infty}-\left.\epsilon\left(x-c_{R} t\right)\right|_{\xi=0, \eta=-\infty} \\
& =\epsilon^{2} \frac{1}{c} \sqrt{\frac{B_{0} u_{0}}{2}}, \\
\Delta_{B} & =\left.\epsilon\left(x+c_{L} t\right)\right|_{\eta=0, \xi=-\infty}-\left.\epsilon\left(x+c_{L} t\right)\right|_{\eta=0, \xi=+\infty} \\
& =-\epsilon^{2} \frac{1}{c} \sqrt{\frac{A_{0} u_{0}}{2}} .
\end{aligned}
$$

Since soliton $A$ is traveling to the right and soliton $B$ is traveling to the left, we see that due to the collision each dark soliton in the condensate has a positive phase shift in its traveling direction. The magnitude of the phase shift for soliton $A(B)$ is proportional to the square root of the greyness $\left[B_{0}\left(A_{0}\right)\right]$ of soliton $B(A)$. This result is at apparent variance with old and recent experiments with collisions and wall reflections (a virtual collision with the corresponding mirror image soliton) of solitons in fluids [19-21,42-44], where a negative phase shift is observed for elevation solitons. In fact, the less usual depression solitons may exhibit positive phase shifts upon collisions [14]. The positive phase shift may be due to the role played by the condensate background in the dynamics of the collision, which becomes dominant upon the actual phase shift occurring asymptotically in the kinematics of the trajectories after collision. Indeed, the soliton velocities are drastically lowered when their amplitudes - depressions of the background pedestal levelincreases. Thus, upon approaching each other, the solitons reduce their velocity, and hence, the trajectory change, in the space-time plot, leading to a positive phase shift. In fluid experiments [42-44], solitons rather accelerate while colliding, and hence, a corresponding negative phase shift occurs. Let us note, for the record and with no further discussion, that there are cases of soliton wall reflection [47] and hence, virtual collisions, as mentioned above, where an expected negative phase shift appears rather as positive, and this can be attributed to the "active" role played by the wall-wave interaction that can be considered as a background effect in the BEC terminology. Thus, we have a clear-cut prediction that if observed would assess the genuine solitonic nature of the condensate density minima as nonlinear, soliton waves.

\section{NUMERICAL RESULTS}

To cross check the above-made statements and extend analytical results into a wider parameter domain, and also to consider the affect of the trapping potential along the axial $(x)$ direction, in this section we numerically investigate the solitonlike solutions of Eqs. (5) and (6).

Before proceeding with the direct numerical integration, for convenience, we change variables

$$
A=\sqrt{\frac{\omega_{x}}{\omega_{\perp} Q}} \rho, \quad t=\frac{2 \omega_{\perp}}{\omega_{x}} \tau, \quad x=\sqrt{\frac{\omega_{\perp}}{\omega_{x}}} s,
$$

where $s$ and $\tau$ are new axial coordinate and time, and $\rho$ is a new variable proportional to the amplitude of the order parameter. Then, Eqs. (5) and (6) are transformed into 


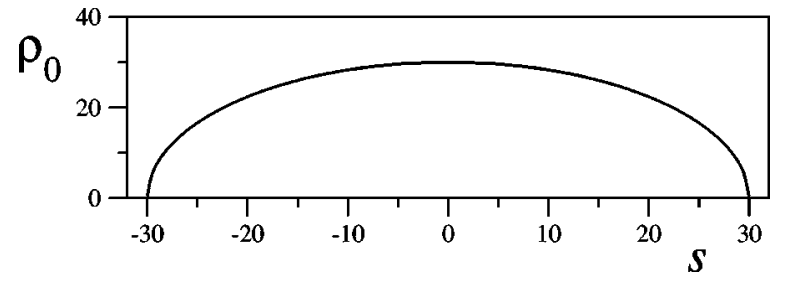

FIG. 1. Stationary background profile [solution of Eqs. (45) with $l=30]$.

$$
\begin{gathered}
\frac{\partial \rho}{\partial \tau}=-2 \frac{\partial \rho}{\partial s} \frac{\partial \varphi}{\partial s}-a \frac{\partial^{2} \varphi}{\partial s^{2}}, \\
\rho \frac{\partial \varphi}{\partial \tau}=\frac{\partial^{2} \rho}{\partial s^{2}}-\left[s^{2}-l^{2}+\rho^{2}+\left(\frac{\partial \varphi}{\partial s}\right)^{2}\right] \rho .
\end{gathered}
$$

The advantage of the new Eqs. (43) and (44) is that they involve one independent constant, $l$, only, where $l$ $=\sqrt{\omega_{x} / \omega_{\perp}} L$, with $L$ being the half length of the condensate. The constant $l$ in Eqs. (43) and (44) plays the role of the problem length. This drastically simplifies the problem for numerical simulations. We assume that the particle number in the condensate is big enough, and hence, on the left and right boundaries the order parameter approximately vanishes, which means that Eqs. (43) and (44) may be supplied by the boundary conditions $\rho( \pm l, \tau)=0$ and $\varphi_{s}^{\prime}( \pm l, \tau)=0$ in the simulations.

As we have seen above, as weakly nonlinear excitations, solitons propagate on some stationary condensate background pedestal $u_{0}$ that is taken to be approximately constant for analytic. Now let us find the shape of this background state $\left[\rho_{0}(s), \varphi_{0}(s)\right]$, when the axial potential is taken into account. Since in the ground state of the system the phase $\varphi_{0}(s)=$ constant, we get from Eqs. (43) and (44) the timeindependent, nonlinear equation for $\rho_{0}$

$$
\frac{d^{2} \rho_{0}}{d s^{2}}=\left[s^{2}-l^{2}+\rho_{0}^{2}\right] \rho_{0} .
$$

We have solved the boundary value problem (45) for a number of different lengths $l=6,8,10,12$, and 30 with the boundary conditions $\rho_{0}( \pm l)=0$. The highest value, $l=30$, corresponds to the approximation used in the theoretical analysis given above, since in this case, the soliton space scale is shorter than the scale of the background state. Besides, this value coincides with the condensate length used by Burger et al. [9]. Figure 1 shows background pedestal profile [solution of Eq. (45)] with $l=30$. Values $l=6-12$ correspond to relatively short condensate lengths or low amount of atoms. We shall use them further to detect the soliton phase shifts due to a head-on collision.

The profiles thus obtained can be used as initial conditions in the numerical integration of Eqs. (43) and (44). All solutions now can be obtained by applying appropriate perturbations to the background pedestal solution.

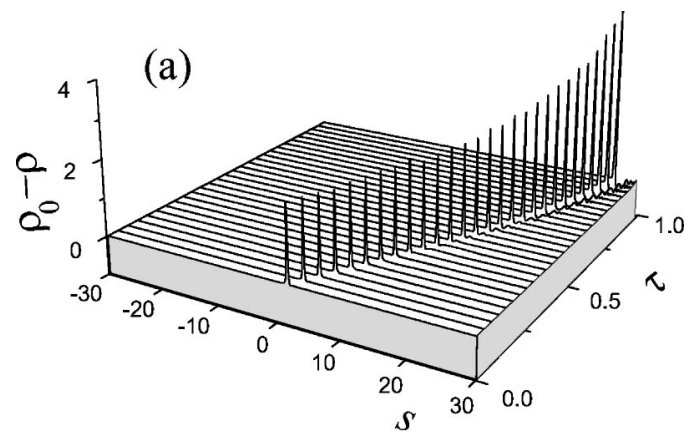

(b)

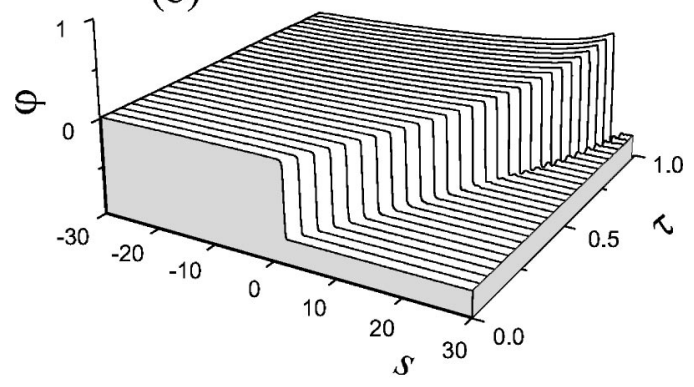

FIG. 2. Space-time plots of a soliton propagating to the right. (a) relative amplitude, (b) phase $\left(\varepsilon=0.07, s_{0}=0\right)$.

Let us start with a test on the single soliton propagation. Taking into account approximate solution (15), (16) and the change of variables (42) we have the following initial conditions

$$
\begin{gathered}
\rho_{1 \text { sol }}(s, 0)=\rho_{0}\left(1-\varepsilon \operatorname{sech}^{2}\left[\sqrt{\varepsilon} \rho_{0}\left(s-s_{0}\right)\right]\right), \\
\varphi_{1 \text { sol }}(s, 0)=-\sqrt{2 \varepsilon} \tanh \left[\sqrt{\varepsilon} \rho_{0}\left(s-s_{0}\right)\right],
\end{gathered}
$$

where $s_{0}$ is an initial soliton position, $\varepsilon$ is a constant responsible for the corresponding amplitude (grayness) of the soliton, and $\rho_{0}$ is the background pedestal solution just found (Fig. 1). We note, however, that the initial conditions given by Eq. (46) are not suitable for direct numerical integration due to the deviation from the background profile near the boundaries, where $\varphi$ must be flat and $\rho$ must approach the background solution $\rho_{0}$. Such perturbations result in nondesired pulses propagating inwards from the boundaries to the center of the interval. To avoid this problem, we correct the initial conditions (46) in a such a way that they approach the stationary background solution near boundaries (far from the initial soliton position $s_{0}$ ), and keep the shape of the soliton (46) in the vicinity of $s_{0}$.

Figure 2 shows results of the numerical integration of Eqs. (43) and (44) providing both amplitude and phase evolution. In Fig. 2(a) we have plotted amplitude of the soliton over the background state, since a direct visualization of $\rho$ is complicated due to its curvature (Fig. 1). The soliton propagates to the right end of the interval. During the propagation the pedestal height $\rho_{0}$ decreases (Fig. 1), while the relative soliton height, $\rho_{0}-\rho$, increases, and hence, the soliton becomes darker, while its velocity decreases. As theory predicts, the phase gradient increases as the soliton approaches the boundary [Fig. 2(b)]. Close to the right boundary, we 

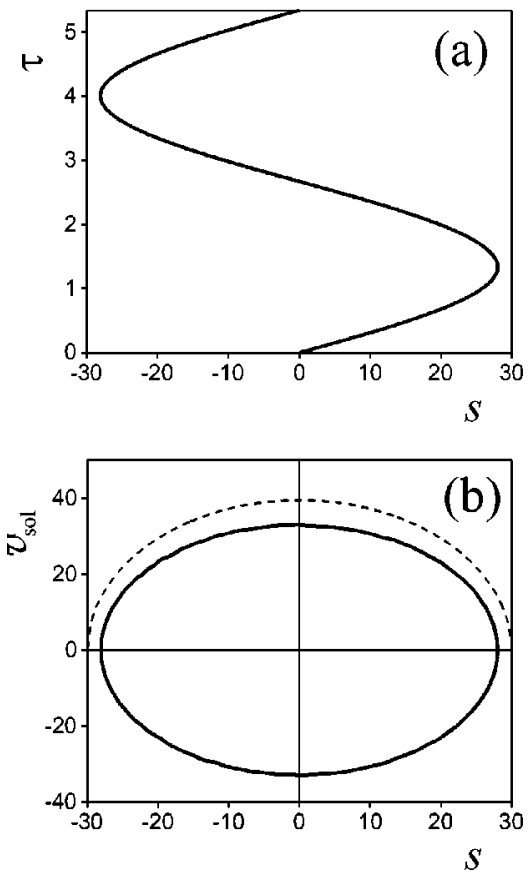

FIG. 3. Soliton "oscillations" between condensate boundaries. (a) Soliton path; (b) Soliton velocity vs $s$ (dashed line corresponds to the theoretically predicted value). Whole cycle corresponds to the time period $\tau \approx 5$.3. At $\tau_{1}^{*} \approx 1.32, s_{1}^{*} \approx 28$ and $\tau_{2}^{*} \approx 4.0, s_{2}^{*} \approx-28$ the soliton velocity changes its sign and the soliton reverse propagation direction. At these instants of time we have $\rho=0$ and $|\Delta \varphi|$ $=\pi$. All parameter values are the same as in Fig. 2 .

have observed a sharp increase of the soliton height and phase difference along soliton, $\Delta \varphi=\varphi_{\text {before }}-\varphi_{\text {after }}$. At certain critical points $\left(\tau_{1}^{*} \approx 1.32, s_{1}^{*} \approx 28\right.$ and $\tau_{2}^{*} \approx 4.0, s_{2}^{*}$ $\approx-28$ in Fig. 3) the soliton approaches the zero overall amplitude, $\rho\left(s_{1,2}^{*}, \tau_{1,2}^{*}\right)=0$, i.e., it becomes plain black. For these instants of time $|\Delta \varphi|=\pi$. Then the phase shift changes its sign, the soliton reveres motion and starts to propagate in the opposite direction with decreasing amplitude and phase shift (Fig. 3). After such "oscillations" between boundaries, at $\tau \approx 5.3$, the soliton comes back to the initial state (Fig. 3). The theoretical estimate for the soliton velocity gives the subsonic velocity

$$
v_{\mathrm{sol}}=\sqrt{2} \rho_{0}(1-\varepsilon)
$$

The soliton velocity is a function of the position coordinate $s$ due to the inhomogeneity along the elongated axis. Figure 3(b) shows the theoretical estimate (47) and data taken from the computer simulation. The numerically found soliton velocity is even lower than Eq. (47). Similar oscillating behavior of the dark solitons in harmonic traps has also been predicted in Ref. [31], but have not yet been observed in experiment. This may be due to the fact that at finite temperature, dark solitons are thermodynamical unstable. The interaction of a soliton with the thermal cloud causes dissipation that accelerates the soliton. In most cases, the soliton reaches the sound speed and disappears before reaching the boundary of the condensate [28]. Thus, to observe this oscil- lation the temperature should be lowered enough to reduce the thermal cloud, and hence, to increase the lifetime of the soliton [9].

Now let us investigate the evolution of two-soliton solutions of the system. We focus on the head-on collision and the theoretically predicted positive phase shift of the solitons after collision. From Eq. (40) the shift of the soliton $A$ (initially left) along the axial coordinate $x$ due to interaction with the soliton $B$ (initially right) is

$$
\Delta x=\sqrt{\frac{\varepsilon_{R}}{Q}},
$$

where $\varepsilon_{R}=\epsilon^{2} B_{0} / u_{0}$ is the initial relative amplitude of the soliton $B$ [analogous to $\varepsilon$ in (46)]. Equation (48) involves the constant $Q$ that depends on the total number of atoms $N$ that, in turn, determines the condensate length $L$ of the system. In Eqs. (43) and (44) we have used the length $l$ as a parameter. To numerically check Eq. (48) a relationship between $Q$ and $L$ is needed.

The solution of Eq. (45) (Fig. 1) can be approximated as

$$
\rho_{0} \approx \sqrt{l^{2}-s^{2}}
$$

Hence, the condensate density corresponding to the stationary background (i.e., ground state) of the system is

$$
n=\frac{\omega_{x}^{2}}{Q \omega_{\perp}^{2}}\left(L^{2}-x^{2}\right) \exp \left[-\frac{y^{2}+z^{2}}{2}\right] .
$$

Using the normalization condition $\int n d \mathbf{r}=1$ we get

$$
Q=\frac{3 \omega_{\perp}^{2}}{4 \pi L^{3} \omega_{x}^{2}}
$$

Thus, from Eq. (48), the estimate for the change of trajectory or phase shift of the soliton $A$ due to head-on collision is

$$
\Delta x=\sqrt{\frac{3 \varepsilon_{R} \omega_{\perp}^{2}}{4 \pi L^{3} \omega_{x}^{2}}},
$$

which yields the phase shift of the soliton $A$ along the coordinate $s$ as

$$
\Delta s \propto \sqrt{\frac{\varepsilon_{R}}{l^{3}}} .
$$

Let us now numerically check the relationship (52). Using Eqs. (36) and (37) we have the following initial conditions

$$
\begin{aligned}
\rho_{2 s o l}(s, 0)= & \rho_{0}\left(1-\varepsilon_{L} \operatorname{sech}^{2}\left[\sqrt{\varepsilon_{L}} \rho_{0}\left(s-s_{L}\right)\right]\right. \\
& \left.-\varepsilon_{R} \operatorname{sech}^{2}\left[\sqrt{\varepsilon_{R}} \rho_{0}\left(s-s_{R}\right)\right]\right), \\
\varphi_{2 s o l}(s, 0)= & \sqrt{2 \varepsilon_{L}}\left(1-\tanh \left[\sqrt{\varepsilon_{L}} \rho_{0}\left(s-s_{L}\right)\right]\right) \\
& +\sqrt{2 \varepsilon_{R}}\left(1+\tanh \left[\sqrt{\varepsilon_{R}} \rho_{0}\left(s-s_{R}\right)\right]\right),
\end{aligned}
$$




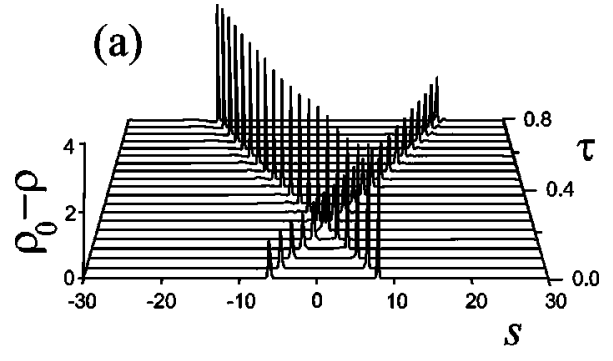

(b)

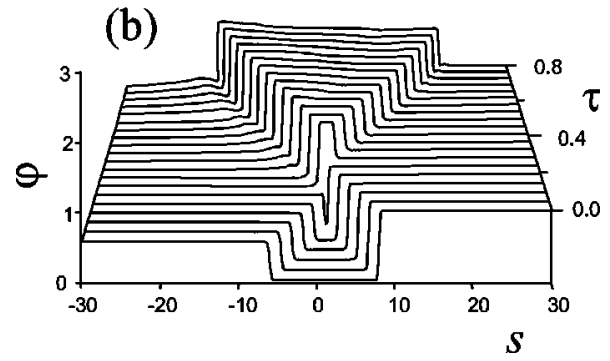

FIG. 4. Space-time plots of two colliding solitons. (a) relative amplitude, (b) phase $\left(\varepsilon_{R}=0.13, \varepsilon_{L}=0.04, s_{L}=-6, s_{R}=8\right.$, and $l$ $=30$ ).

where $s_{L}$ and $s_{R}$ are initial positions of the left $(A)$ and the right solitons $(B)$, respectively, and $\varepsilon_{L}, \varepsilon_{R}$ are their "amplitudes.' Again, like in the case of one soliton, we have appropriately corrected at the beginning of computation the solution (53) to get well-shaped solitons.

Figure 4 shows results of the experimental test. Two solitons propagate in opposite directions, approach each other, collide and, asymptotically, separate away. During collision, one practically motionless composite structure forms for some time interval (actually, it is a depression of the background pedestal level). Its velocity depends on the initial soliton heights. For identical solitons such composite structure does not move, but changes its shape. Due to the relatively long length $l$ we cannot observe any remarkable phase shift in this experiment. Thus, in accordance to Eq. (52), to observe and measure the positive phase shift predicted by the theory we reduce the length to $l=6-12$ and make new computer experiments.

We have found two different cases of head-on soliton collision (Fig. 5):

(i) 'Gray collision', [Fig. 5(a,c)]. If the initial soliton amplitudes or depression depths are small enough $\left(\varepsilon_{R}=\varepsilon_{L}\right.$ $<0.6$ for $l=8$ ), then during collision, a single composite structure forms and further increases its amplitude but never touches zero, i.e., waves remain always gray. Figure 5(c) shows the paths of the corresponding solitons. Clearly, around $\tau=0.2$, the solitons form a single composite solution, which survives during some time interval (vertical bar linking two paraboliclike paths). Then, the solitons separate away.

(ii) "Black collision" [Fig. 5(b,d)]. If the initial soliton amplitudes are large enough, then, while the solitons approach each other, their amplitudes grow and at some instant of time, just before collision, the soliton amplitudes vanish as their corresponding depressions touch zero (solitons become plain black) still remaining on some distance from each other [fourth snapshot on Fig. 5(b)]. Thus, in this case, the solitons
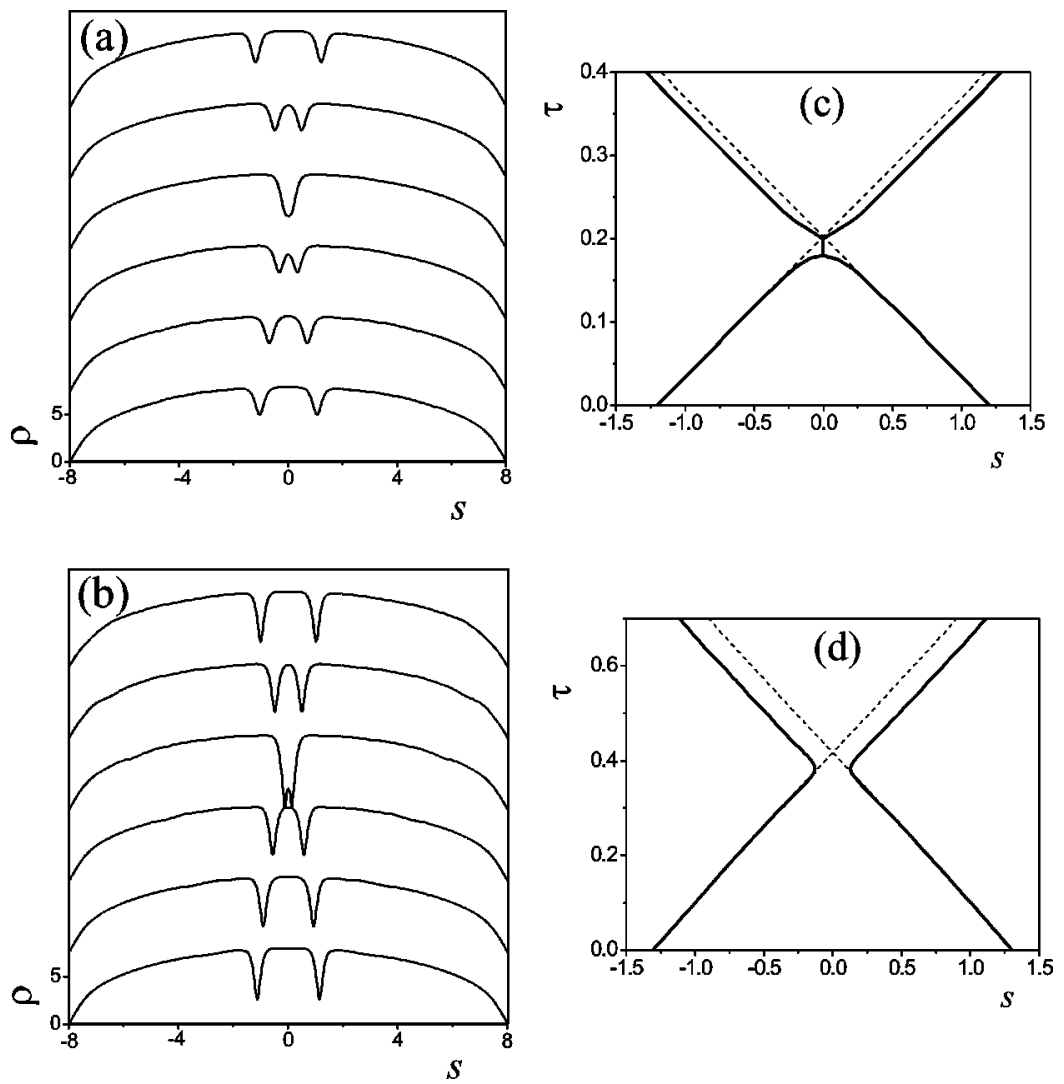

FIG. 5. Two different types of soliton collisions. (a,c) Gray collision $\left(\varepsilon_{R}=\varepsilon_{L}=0.4\right)$; (b,d) Black collision $\left(\varepsilon_{R}=\varepsilon_{L}=0.75\right)$. (a,b) Sequential snapshots of amplitude distribution, (c, d) soliton paths (only a piece, $s \in[-1.5,1.5]$, of the whole interval is plotted). Dotted lines mark soliton path as if each of them would propagate alone. 


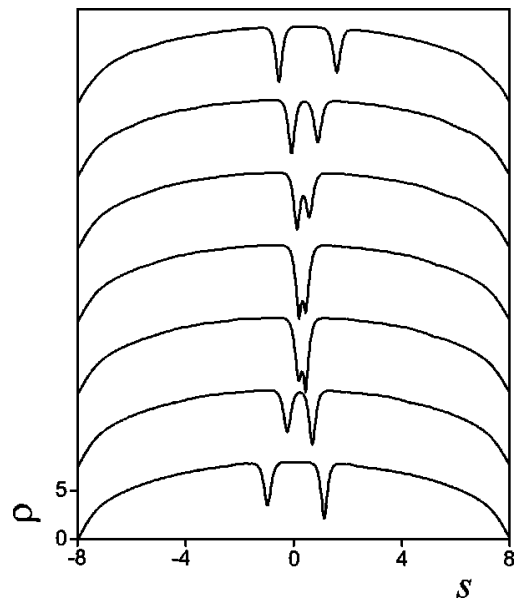

FIG. 6. Black collision of two solitons of different amplitude. $\left(\varepsilon_{R}=0.6, \varepsilon_{L}=0.8\right)$.

never form one hump solution [Fig. 5(d)]. This looks similar to the reflection from the boundary described above. However, during collision, solitons exchange energy, hence, in the case of solitons with different heights, the propagation directions are preserved (Fig. 6), i.e., the small soliton after collision continues to propagate to the right, while the big one runs to the left.

Clearly, Fig. 5(c) and 5(d) show positive soliton shifts in their own traveling directions after the collisions. Let us now measure $\Delta s$ as a function of $\varepsilon^{1 / 2}$ and $l^{-3 / 2}$ and compare with the theoretically predicted relationship (52) (Fig. 7). The
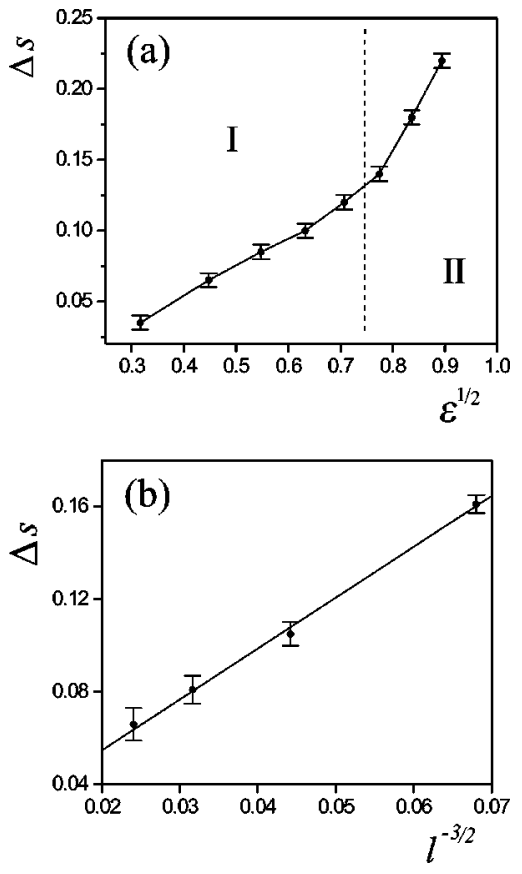

FIG. 7. Axial soliton shift $\Delta s$ as a function of (a) initial relative soliton amplitude, $\varepsilon^{1 / 2}\left(l=8, \varepsilon_{R}=\varepsilon_{L}=\varepsilon\right)$, and (b) of the length $l^{-3 / 2}(\varepsilon=0.4)$. curve on Fig. 7(a) has two regions. Region I corresponds to the dark type of collision (for which the theory has been developed). In this region the experimental data can be well fitted with a straight line, that confirms the correctness of the relationship (52). In the region II (high soliton amplitude) the effect of the interaction with the "black ground" (zero amplitude) plays a significant role, and hence, $\Delta s$ grows much faster. Figure 7(b) shows results of experiments with the same $\varepsilon_{R}$ but varying $l$. The value of $\varepsilon_{R}$ was taken from the region I [Fig. 7(a)]. Again, the data fits well on a straight line, hence confirming well the relationship (52). We note that, because of the inhomogeneity along the elongated axis, the soliton paths will bend near the boundary of the condensate background as we have seen in Fig. 3 for the single soliton solution.

\section{DISCUSSION AND SUMMARY}

We have investigated the evolution of weakly nonlinear pulses in a cigar-shaped BEC with a repulsive interaction. A consistent description using a Boussinesq-Korteweg-de Vries (B-KDV) evolution equation for dark soliton propagation has been developed without using the Thomas-Fermi (TF) approximation. We emphasize that the dispersion provided by the quantum pressure in the condensate plays a significant role in the formation of the dark solitons. The head-on collision between two dark solitons traveling in opposite directions has also been investigated by means of the Poincare-Lighthill-Kuo (PLK) method. The analytical and computer experiment results show that due to the collision each soliton displays a positive phase shift in its traveling direction, whose magnitudes we have obtained. The corresponding trajectory change is at variance with known results in fluids dynamics [19-21] but we have shown that in the BEC case the background plays a dominant role in the collision events, which is not the case in experiments with fluids, unless we consider the less usual case of depression solitons [14,42-44].

In the approach presented in Ref. [35], a TF approximation for the ground- and also for the excited-state is made, which disregards the quantum pressure, and hence, the dispersion of the system is neglected. Furthermore, the assumption was made of an "interface" for the condensate, taken as an elastic cylindrical shell and obeying Newton's second law. Using this assumption, a new equation, not resulting from the Gross-Pitaevskii (GP) equation, was introduced. The dispersion of the B-KdV equation obtained in Ref. [35] follows from this new equation with, however, a different sign relative to that obtained when the dispersion originates from the quantum pressure of the system. This is the reason why, in Ref. [35], a bump ("bright") soliton with its propagating velocity higher than the sound speed of the system was obtained. In our approach, the TF approximation is not used. The dark solitons form due to the balance between the quantum pressure-driven dispersion and the nonlinearity in the condensate and we obtain dark, subsonic solitons.

The results presented in this paper can be tested by experiment. For a long enough cigar-shaped condensate with a repulsive atom-atom interaction, one can apply simulta- 
neously two far off-resonant laser fields to the condensate as phase imprinting at two different positions, say at a point $A$ and at a point $B$ in the elongated axis direction. At each point, a density minimum (dark soliton) can be generated propagating towards each other in opposite directions. Then, a head-on collision between two dark solitons is expected to happen and the phase shifts following the collision can be obtained following in a space-time plot their corresponding trajectories before and after the collision. Note that considering the finite soliton lifetime at finite temperatures (due to the interaction of the soliton with the thermal cloud), the distance between points $A$ and $B$ should be short enough to ensure that they survive long to experience the head-on collision.

\section{ACKNOWLEDGMENTS}

The authors wish to express their gratitude to V. V. Konotop for enlightening discussions. V.A.M. benefited from financial support from the Spanish Ministry of Education and Culture. This research was supported by grants from the Natural Science Foundation of China, from the TransCentury Training Program for the Talents of the Ministry of Education of China, and from the Spanish Ministry of Science and Technology (PB 96-599).
[1] M. H. Anderson, J. R. Ensher, M. R. Matthews, C. E. Wieman, and E. A. Cornell, Science 269, 198 (1995).

[2] K. B. Davis, M.-O. Mewes, M. R. Andrews, N. J. van Druten, D. S. Durfee, D. M. Kurn, and W. Ketterle, Phys. Rev. Lett. 75, 3969 (1995).

[3] C. C. Bradley, C. A. Sackett, J. J. Tollett, and R. G. Hulet, Phys. Rev. Lett. 75, 1687 (1995); 78, 985 (1997).

[4] D. G. Freid, T. C. Killian, L. Willmann, D. Landhuis, S. C. Moss, D. Kleppner, and T. J. Greytak, Phys. Rev. Lett. 81, 3811 (1998).

[5] A. S. Parkins and D. F. Walls, Phys. Rep. 303, 1 (1998).

[6] F. Dalfovo, S. Giorgini, L. P. Pitaevskii, and S. Stringari, Rev. Mod. Phys. 71, 463 (1999).

[7] The reference list on the BEC homepage http:// amo.phys.gasou.edu/bec.html

[8] M. R. Mattews, B. P. Anderson, P. C. Haljan, D. S. Hall, C. E. Wieman, and E. A. Cornell, Phys. Rev. Lett. 83, 2498 (1999).

[9] S. Burger, K. Bongs, S. Dettmer, W. Ertmer, and K. Sengstock, Phys. Rev. Lett. 83, 5198 (1999).

[10] J. Denschlag, J. E. Simsarian, D. L. Feder, C. W. Clark, L. A. Collins, J. Cubizolles, L. Deng, E. W. Hagley, K. Helmerson, W. P. Reinhart, S. L. Rolston, B. I. Schneider, and W. D. Phillips, Science 287, 97 (2000).

[11] L. Deng, E. W. Hagley, J. W. En, M. Trippenbach, Y. Band, P. S. Julienne, J. E. Simsarian, K. Helmerson, S. L. Rolston, and W. D. Phillips, Nature (London) 398, 218 (1999).

[12] A. R. Bishop and T. Schneider, Solitons in Condensed Matter Physics (Springer, Berlin, 1978).

[13] A. D. D. Craik, Wave Interactions and Fluid Flows (Cambridge University, Cambridge, England, 1985).

[14] A. A. Nepomnyashchy and M. G. Velarde, Phys. Fluids 6, 187 (1994).

[15] C. I. Christov and M. G. Velarde, Physica D 86, 323 (1995).

[16] A. C. Newell and J. V. Moloney, Nonlinear Optics (Addison Wesley, Red Wood, CA, 1992).

[17] Y. S. Kivshar and B. A. Malomed, Rev. Mod. Phys. 61, 763 (1989).

[18] Y. S. Kivshar and B. Luther-Davies, Phys. Rep. 298, 81 (1998).

[19] J. W. Miles, J. Fluid Mech. 79, 157 (1977); 79, 171 (1977).

[20] M. G. Velarde, A. A. Nepomnyashchy, and M. Hennenberg, Adv. Appl. Mech. 37, 167 (2000).
[21] P. G. Drazin and R. S. Johnson, Solitons: An Introduction (Cambridge University, Cambridge, England, 1989).

[22] W. P. Reinhart and C. W. Clark, J. Phys. B 30, L785 (1997).

[23] A. D. Jackson, G. M. Kavoulakis, and C. J. Pethick, Phys. Rev. A 58, 2417 (1998).

[24] R. Dum, J. I. Cirac, M. Lewenstein, and P. Zoller, Phys. Rev. Lett. 80, 2972 (1998).

[25] T. Hong, Y. Z. Wang, and Y. S. Huo, Phys. Rev. A 58, 3128 (1998).

[26] T. F. Scott, R. J. Ballagh, and K. Burnett, J. Phys. B 31, L329 (1998).

[27] A. E. Muryshev, H. B. van Linden van den Heuvell, and G. V. Shlyapnikov, Phys. Rev. A 60, R2665 (1999).

[28] P. O. Fedichev, A. E. Muryshev, and G. V. Shlyapnikov, Phys. Rev. A 60, 3220 (1999).

[29] L. Dobrek, M. Gajda, M. Lewenstein, K. Sengstock, G. Birkl, and W. Ertmer, Phys. Rev. A 60, R3381 (1999).

[30] D. Margetis, J. Math. Phys. 40, 5522 (1999).

[31] Th. Busch and J. R. Anglin, Phys. Rev. Lett. 84, 2298 (2000).

[32] D. L. Feder, M. S. Pindzola, L. A. Collins, B. I. Schneider, and C. W. Clark, Phys. Rev. A 62, 053606 (2000).

[33] L. D. Carr, C. W. Clark, and W. P. Reinhardt, Phys. Rev. A 62, 063610 (2000).

[34] L. D. Carr, M. A. Leung, and W. P. Reinhardt, J. Phys. B 33, 3983 (2000).

[35] T. Tsurumi and M. Wadati, J. Phys. Soc. Jpn. 67, 2294 (1998).

[36] C. I. Christov, G. A. Maugin, and M. G. Velarde, Phys. Rev. E 54, 3621 (1996).

[37] A. Jeffery and T. Kawahawa, Asymptotic Methods in Nonlinear Wave Theory (Pitman, London, 1982).

[38] V. M. Perez-Garcia, H. Michinel, and H. Herrero, Phys. Rev. A 57, 3837 (1998).

[39] G. M. Kavoulakis and C. J. Pethick, Phys. Rev. A 58, 1563 (1998).

[40] M. R. Andrews, D. M. Kurn, H.-J. Miesner, D. S. Durfee, C. G. Townsend, S. Inouge, and W. Ketterle, Phys. Rev. Lett. 79, 553 (1997); 80, 2967 (1998).

[41] N. J. Zabusky and M. D. Kruskal, Phys. Rev. Lett. 15, 240 (1965).

[42] H. E. Crossley, Jr., CALTECH Report No. N-54.1, 1949 (unpublished).

[43] C. H. Su and R. M. Mirie, J. Fluid Mech. 98, 509 (1980). 
[44] H. Linde, X.-L. Chu, and M. G. Velarde, Phys. Fluids A 5, 1068 (1993).

[45] Guoxiang Huang, Senyue Lou, and Zaixin Xu, Phys. Rev. E 47, R3830 (1993).
[46] Guoxiang Huang, M. G. Velarde, and V. N. Kurdyumov, Phys. Rev. E 57, 5473 (1998).

[47] H. Linde, M. G. Velarde, W. Waldhelm, and A. Wierschem, J. Colloid Interface Sci. 236 (in press). 